\title{
Design Alternatives for a Thin Lens Spatial Integrator Array
}

\author{
Hala Kamal', Daniel V'azquez and Javier Alda and E. Bernabeu \\ Optics Department. University Complutense of Madrid. \\ School of Optics. Av. Arcos de Jal'on s/n. 28037 Madrid. Spain.
}

\begin{abstract}
In this present paper, we present a theoretical analysis relating the type, power and the maximum number of the identical elements in the input surface of the array, with its size, flux transfer efficiency onto the synthetic image plane and the synthetic image quality. The numerical analysis results enabled the design of two different planar thin-lens spatial integrator arrays with optimized geometrical parameters and with different positive and negative power values. A computer program has been developed to investigate the performance of each array. The percentage of the outgoing rays, the average transmittance of the array elements, the image width and the uniformity of the transferred energy, have been evaluated and compared.
\end{abstract}

\section{1- Introduction:}

Lens arrays, formed either by refractive or diffractive lenses, are increasing in importance because they have a lot of new uses in modern aspects of optics. They have aroused considerable interest in the area of controlling beams by allowing individual elements to have their own focusing and directing elements. Compared with a conventional optical system, an optical array has the advantage of working in parallel; it divides the incident wave front into portions which proceed through the individual optical elements to form a composed image. This composed image is called synthetic image and generally it doesn't coincide with the image of the object given by the individual elements. This study is focused on an interesting type of optical arrays called spatial integrators.

\footnotetext{
* On leave From Physics Department, Faculty of Science, Ain Shams University, Cairo, Egypt.
} 
They are non-imaging devices with zero ray transfer matrix determinant, so they are called arrays with vanishing determinant. The essential characteristics of such arrays are, having infinite field depth and an image size independent of the position of the object. In other words, all sources in the object space are imaged at the same plane. Despite of being non imaging devices, they found other several applications. They are used to transfer radiation from an extended source to a target in such a way as to achieve a specified distribution of radiation on the target. They are employed as energy concentrators in solar furnaces and as illuminators in shadow-less lamps.

Within the matrix optics frame work [1, 2], the first design condition for a light integrator array is that the distance $R$ between the input plane and output plane of the array should obeys the following formula

$$
R=l-\frac{b}{d}
$$

where $l$ is the length of the individual optical element, and $b$ and $d$ are the elements of the matrix of each individual element of the array.

In previous research, [3, 4, 7], we have developed several ideas to improve the efficiency for these kinds of arrays. For a spherical thick lens spatial integrator arrays the design parameters are optimized in such a way to transfer as much as possible the energy incident on the array input plane to the synthetic image plane. Also, a planer arrangement was proposed [5,.6, 7] which is easier to be fabricated and integrated into the optical systems.

The purpose of this paper is to explore the influence of the type, power of the array optical elements and the maximum number of array elements on the input surface of the array, on the transferred flux and the image quality formed onto the synthetic image plane (S.I.P.). Planer thin-lens light integrator arrays are studied. The first section deals with a detailed theoretical analysis which explore the influence of the type of the optical element (diverging or converging) in the input surface of the array, its power and the maximum number of identical elements of the array that can work together, in order to transfer the incident flux onto the S.I.P., regardless the individual efficiency of each array element. Secondly, a numerical analysis was done based on the theoretical relations, obtained in the previous section, giving rise to different results describing, both the behavior of a planer thin-lens light integrator array and the behavior of the individual element at different power values. Thirdly, the theoretical predictions as well as the numerical results obtained previously, allows the design of two different thin-lens planar light integrator arrays. Performance as well as comparison of some major aspects of the two designed arrays are given in the fourth section. 


\section{2- Planar Thin lens Spatial Integrators:}

A scheme of a planer thin lens spatial integrator array is shown in Fig.(1). It is composed of a pair of planar thin lens arrays (PTLA) separated by a distance $t$; the pitch of the input plane (PTLA1) is longer than that of the output plane (PTLA2). The individual element of the array is composed of a couple of thin lenses of different focal lengths $f_{1}$ and $f_{2}$. The optical axis of each element is the line joining the centers of the optical elements forming the unit. Each element has its own optical axis, all intersects at the center of the synthetic image plane. The type of the elements on PTLA1 could be either converging, divergent or both lenses

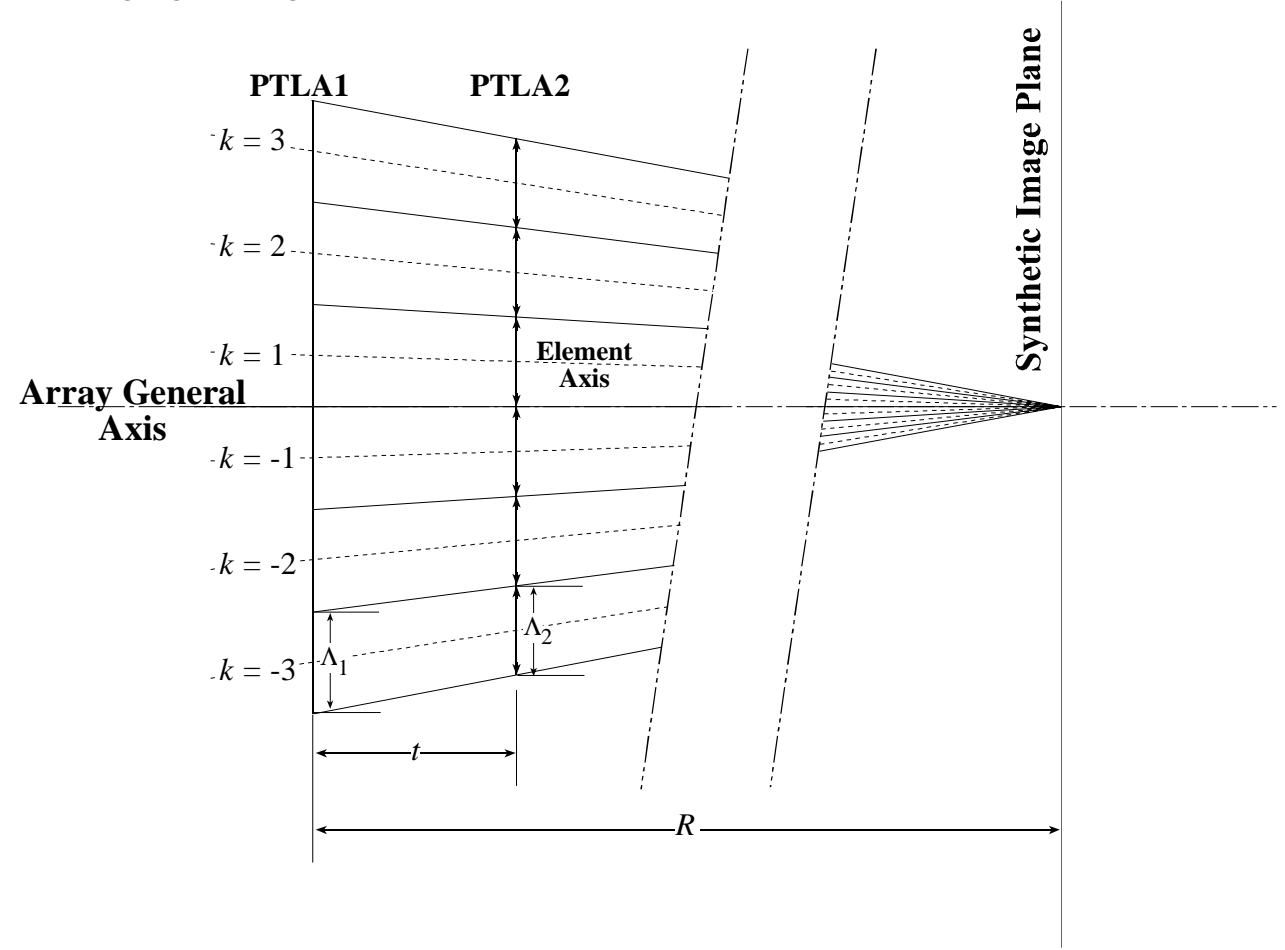

Fig. (1): A light integrator array composed of a couple of planar thin lens arrays (PTLA1 \&PTLA2).

It follows from equation (1) that, for a thin-lens light integrator array, the distance between the input plane and the synthetic image plane of the array is,

$$
R=t\left(1-\frac{f_{2}}{f_{2}-t}\right)
$$


The last equation indicates that the spatial integration feature of this type of arrays doesn't depend on the focal length of the first thin lens $f_{1}$ which is left as a free parameter in the design of the array. Instead, the value of $f_{2}$ and $t$ should be fixed. Very recently [8], the focal length values for PTLA1 optical elements are optimized in such a way to maximize the transfer of light energy incident upon it to the synthetic image plane (S.I.P.). Another criterion that might be taken into account, is the maximum number of elements that can work together in order to transfer light-rays to the synthetic image plane for a given value of $f_{1}$. In this case, we assume that all the elements of PTLA1 have equal focal length $f_{1}$ and we take into consideration all elements that permit the transfer of any part of light-rays incident upon it to the synthetic image plane.

For the planer thin lens-array configuration given in Fig. (1), as the axis of the individual elements become more and more misaligned, the efficiency of the elements to transfer radiation to the synthetic image plane becom smaller. In the following we obtain the maximum order of the elements that a planer thin-lens spatial integrator array can bear, two different cases are considered.

\section{Case (1) : Positive planer input array:}

In this case we assume that, PTLA1 is composed of converging thin lenses of equal focal length $f_{1}$ and the elements of PTLA2 are of identical focal length $f_{2}$, in order to comply with the vanishing determinant condition of equation (2). PTLA1 and PTLA2 are assumed to be of different pitch $\Lambda_{1}$ and $\Lambda_{2}$ respectively, and $\Lambda_{1}>\Lambda_{2}$.

For positive PTLA1 with $f_{1}$ values greater than the individual element size $t$. The maximum order that the individual element can attain is defined by the ray incident at the lower extreme of the $k^{\text {th. }}$ element of PTLA1 and after refraction goes through the upper extreme of the element in PTLA2, Fig. (2)a. Considering a parallel bundle of rays aligned with the array general axis, this can be expressed in a matricial form as;

$$
\left(\begin{array}{c}
-\frac{\Lambda_{1}}{2} \\
\omega_{k}
\end{array}\right)=\left(\begin{array}{cc}
1 & 0 \\
-\frac{1}{f_{1}} & 1
\end{array}\right)\left(\begin{array}{c}
-\frac{\Lambda_{1}}{2} \\
0
\end{array}\right)
$$

where, 


$$
\omega_{k}=\frac{\Lambda_{1}-k\left(\Lambda_{1}-\Lambda_{2}\right)}{t}
$$

The maximum order of the element for $f_{1}>t$;

$$
k=\operatorname{Pr} \text { eviousInteger }\left(\frac{\Lambda_{1}\left(2 f_{1}-t\right)}{2 f_{1}\left(\Lambda_{1}-\Lambda_{2}\right)}\right)
$$

Similarly, for positive PTLA1 with $f_{1}$ values smaller than the individual element size $t$. The maximum order that the individual element can be calculated by considering the light ray incident at the maximum height of the $k^{\text {th. }}$ element of PTLA1 to be deviated with an angle $\theta_{k}$, Fig. (2-b). Considering a parallel bundle of rays aligned with the array general axis, this can be written in a matricial form as;

$$
\left(\begin{array}{c}
\frac{\Lambda_{1}}{2} \\
-\theta_{k}
\end{array}\right)=\left(\begin{array}{cc}
1 & 0 \\
-\frac{1}{f_{1}} & 1
\end{array}\right)\left(\begin{array}{c}
\frac{\Lambda_{1}}{2} \\
0
\end{array}\right)
$$

where,

$$
\theta_{k}=\frac{k\left(\Lambda_{1}-\Lambda_{2}\right)}{t}
$$

The maximum order of the element for $f_{1}<t$;

$$
k=\operatorname{Pr} \text { eviousInteger }\left(\frac{t \Lambda_{1}}{2 f_{1}\left(\Lambda_{1}-\Lambda_{2}\right)}\right)
$$

\section{Case (2) : Negative planer input array}

For a negative planer spatial integrator array, PTLA1 is assumed to be composed of diverging thin lenses of focal length $-f_{1}$, all stacked side by side in a planer arrangement and separated from PTLA2 by a distance $t$. For $\Lambda_{1}>\Lambda_{2}$, the light-ray that defines the maximum number of elements of the array is that which touches the $k^{\text {th. }}$ element of PTLA1 at $-\frac{\Lambda_{1}}{2}$ and then 
deviates by an angle $\varphi_{k}$ where it passes through the corresponding element on PTLA2, Fig.(2)c. Considering a parallel bundle of rays aligned with the array general axis we get;
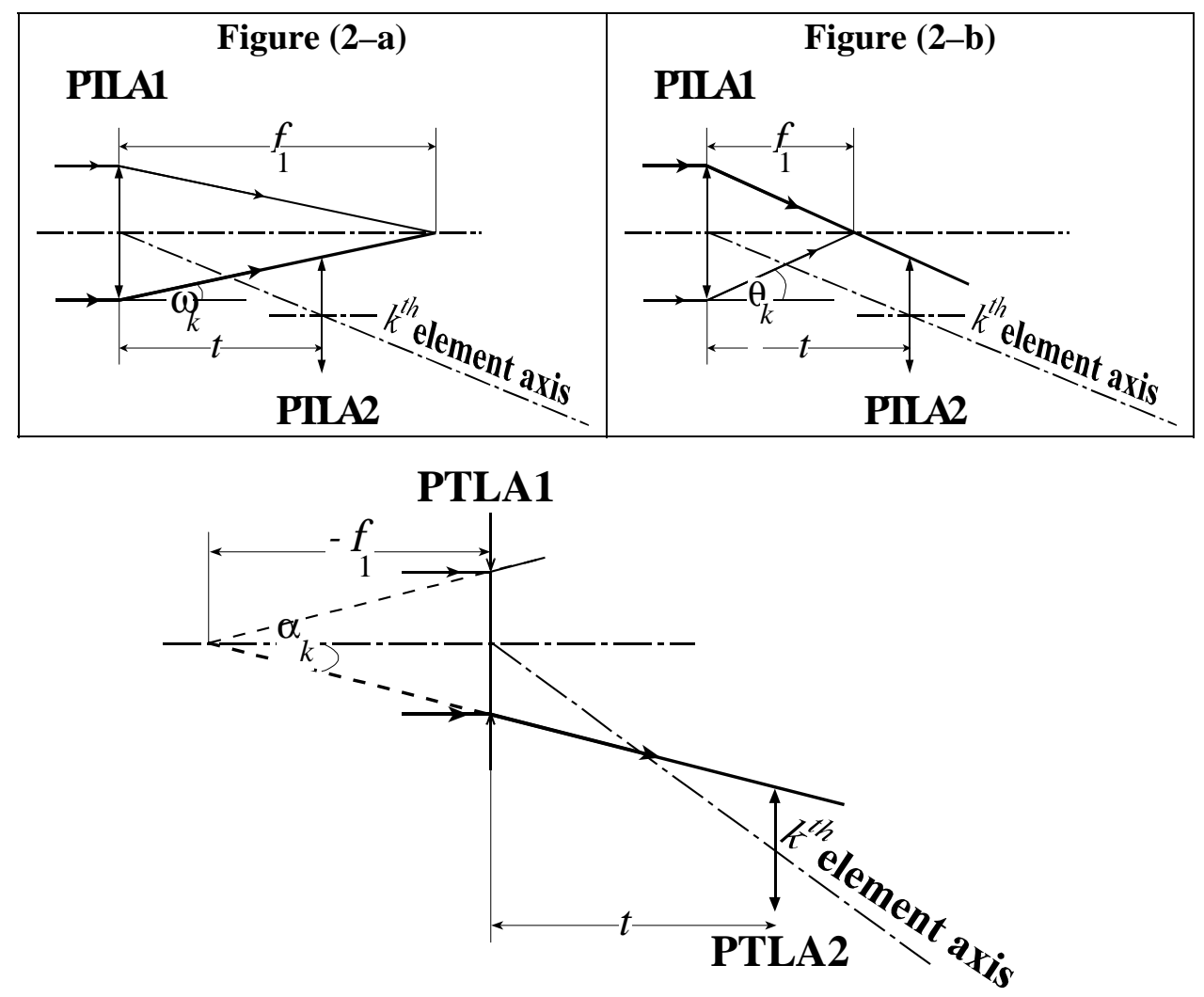

Fig. (2): Scheme of a light integrator array element composed of a couple of thinlenses, the output lens has a positive focal length $f_{2}$. The input lens has, (a) Positive focal length $f_{1}>t$. (b) Positive focal length $f_{1}<t$. (c) Negative focal length.

$$
\left(\begin{array}{c}
-\frac{\Lambda_{1}}{2} \\
-\alpha_{k}
\end{array}\right)=\left(\begin{array}{ll}
1 & 0 \\
\frac{1}{f_{1}} & 1
\end{array}\right)\left(\begin{array}{c}
-\frac{\Lambda_{1}}{2} \\
0
\end{array}\right)
$$

where;

$$
\alpha_{k}=\frac{k\left(\Lambda_{1}-\Lambda_{2}\right)-\Lambda_{1}}{t}
$$


The maximum order of the element is given by;

$$
k=\operatorname{Pr} \text { eviousInteger } \frac{\Lambda_{1}\left(t+2 f_{1}\right)}{2 f_{1}\left(\Lambda_{1}-\Lambda_{2}\right)}
$$

Note that the maximum number of elements of the array is twice the maximum order of the element $k$, as the elements are arranged up and down the array general axis.

\section{Simulation and Results:}

In order to explain and clarify the importance of the Previously obtained relations, we have investigated the behavior of thin lens spatial integrator arrays with maximum number of elements with different positive and negative PTLA1 power values. The input planar thin lens array (PTLA1) is assumed to be of identical elements. A computer program has been developed to independently estimate the behavior of each array. The geometrical parameters of the array used in the computer simulation are given in Table (1), The values have been chosen according to light integrator arrays optimization theory presented recently in [8]. In Fig. (3), we have plotted the power of PTLA1 in diopters (D) versus: (a)The maximum order of the element obtained from the above formulas, (b) The percentage of the outgoing rays developed from each array with respect to the incoming rays, (c) The average transmittance of all array elements, (d) The image width $(\varpi)$ and the coefficient of uniformity $(u)$ for the energy distribution on the synthetic image plane $\phi(x)$, defined as

$$
u=\int_{\text {paraxial }}\left|\frac{\partial \phi(x)}{\partial(x)}\right| d x
$$

Note that, the integration is taken only on the spatial region of the paraxial synthetic image plane. This parameter equals zero for a perfect uniform distribution within the paraxial synthetic image. It is clear from the results depicted in Fig.(3)d that, although the uniformity of the image varies significantly with the power of the individual elements in PTLA1, the image size obtained from the numerical simulation changes slightly which coincides with the theoretical predictions of the paraxial size of the synthetic image $\Delta$, reported previously [1],

$$
\Delta=\frac{\Lambda_{1}}{\left(1-\frac{t}{f_{2}}\right)}
$$


Table (1): Geometrical parameters of a planer thin-lens light integrator array used in the computer simulation. The refractive index of the lenses material is 1.523 .

\begin{tabular}{|c|c|}
\hline $\mathrm{R}$ & $1280 \mathrm{~mm}$ \\
\hline$f_{2}$ & $137.9 \mathrm{~mm}$ \\
\hline$\Lambda_{1}$ & $80 \mathrm{~mm}$ \\
\hline$\Lambda_{2}$ & $70 \mathrm{~mm}$ \\
\hline$t$ & $160 \mathrm{~mm}$ \\
\hline
\end{tabular}
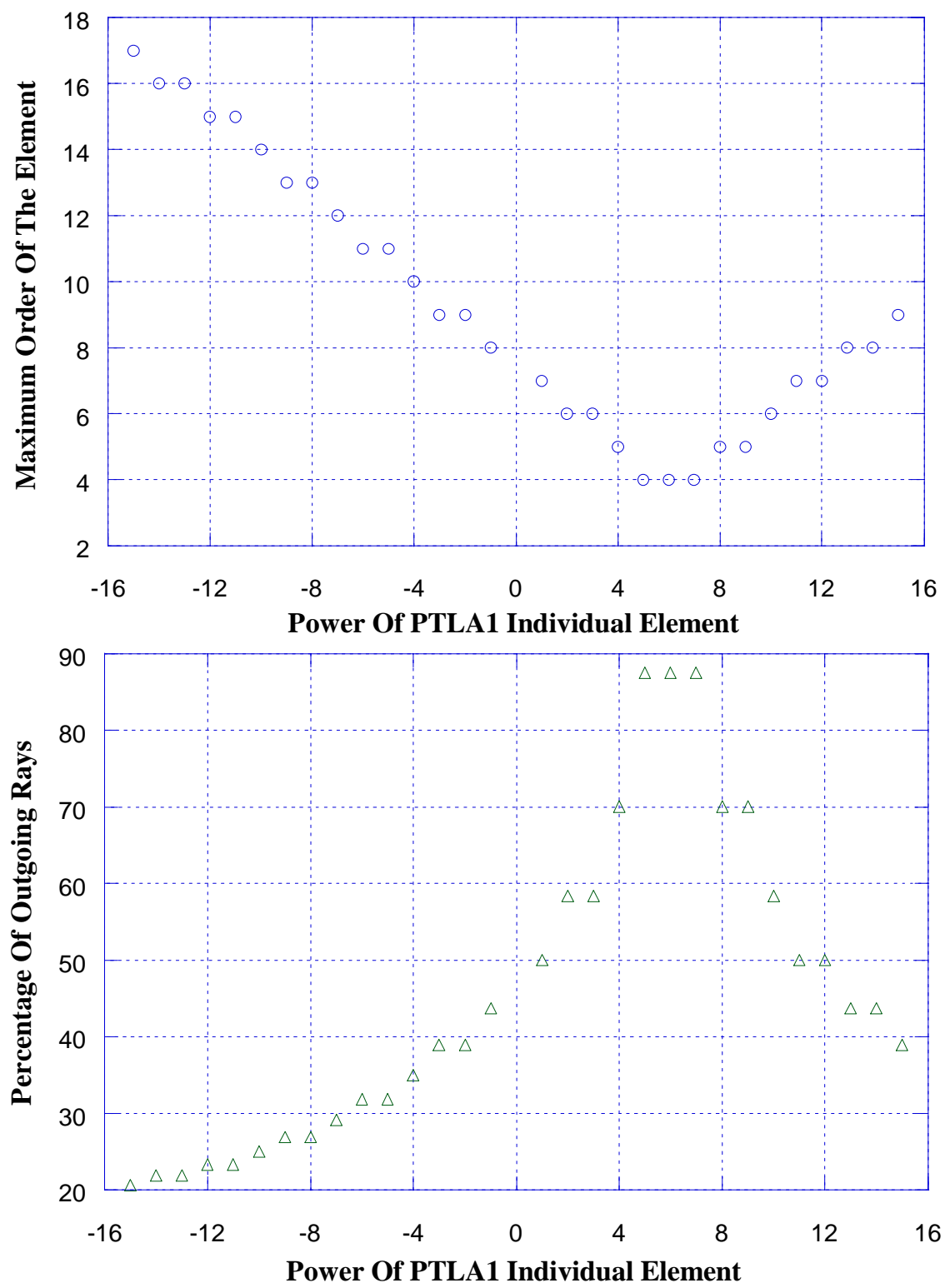


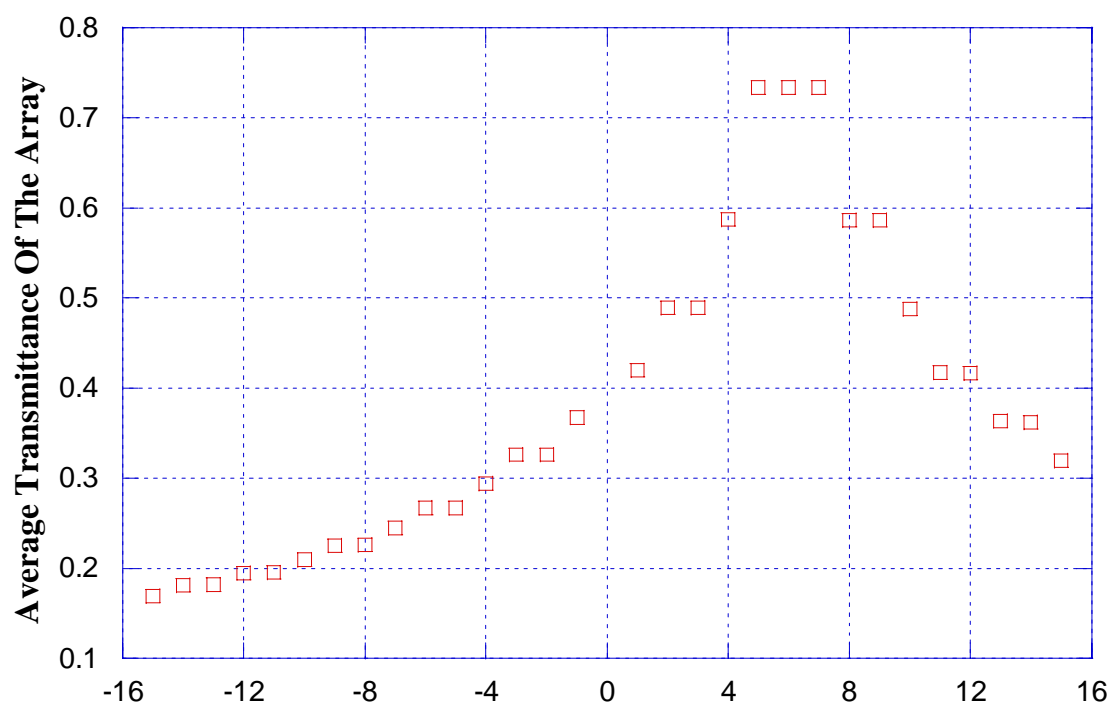

Power Of PTLA1 Individual Element

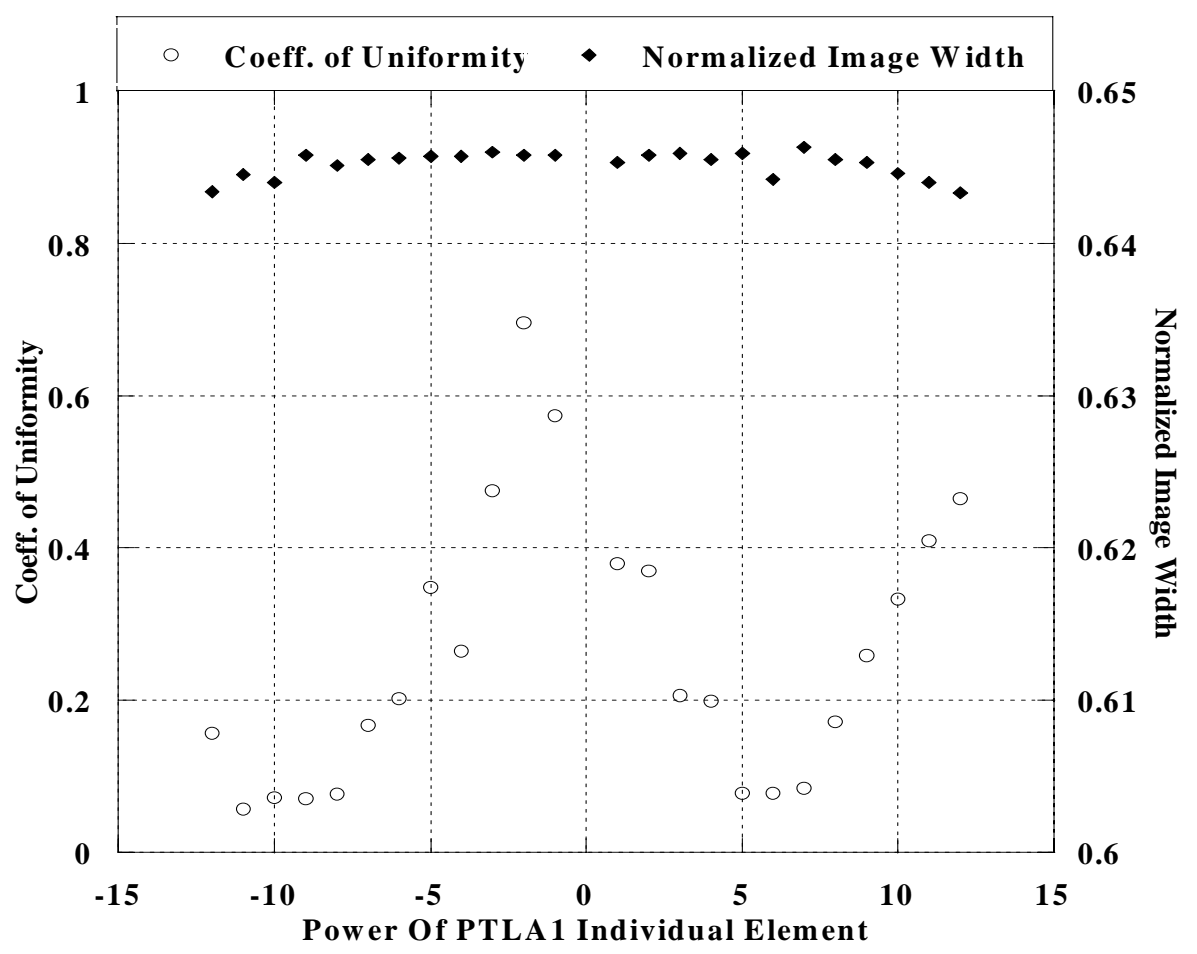

Fig. (3): Relation between the power of PTLA1 elements Versus: (a)The maximum order of the element. (b) The percentage of the outgoing rays. (c) The average transmittance of the array. (d) The image width $(\varpi)$ and the coefficient of uniformity $(u)$. 
Also, the behavior of each array element at different focal length values has been found whereas the power $\left(F_{1}\right)$ of PTLA1 have taken different positive and negative values, namely; $\pm 1, \pm 2, \pm 3, \ldots \ldots \ldots \pm 15$ diopters (D). As a measure of efficiency of each individual element, the percentage of the outgoing rays with respect to the incoming ones as well as the average transmission of the array have been calculated for different power values and depicted in Fig. (3) $\mathrm{b}$ and $\mathrm{c}$. Both curves show peaks at power range 5-7 D for the PTLA1 individual elements. For clarity, the number of the outgoing light-rays with respect to the incident rays has been computed at different order of the element and represented in Fig.(4). It shows that all curves converge at a particular order of the element then diverge again.

The advantages of the previously obtained graphs is that, it enables the design of different spatial integrator arrays adequate for different purposes. In other words, these graphs can be used as a guide in arrays design. In the following, with the aid of the obtained results, design of two different spatial integrator arrays is given. Although, in the previous graphs the power of thin lenses in PTLA1 has attained large values, in designing the following two arrays we have considered thin lenses in PTLA1 with stop number given by;

$$
F_{1}^{\text {number }}=\frac{f_{1}}{\Lambda_{1}}
$$

smaller than two as they are easier to be fabricated).

\section{Design:}

Light integrator arrays have several applications leading to different kinds of arrays. Although, each kind depends on the details of the optical system, some criterion could be laid down which enable defining the global behavior of the array. Those criterian comprise;

(a) Number of array elements (size of the array).

(b) Type of elements on the array input surface (for spatial integrator arrays the optical elements of the input surface doesn't affect the integration feature).

(c) Efficiency of each element.

(d) The uniformity of the image on the synthetic image plane and formed by all array elements.

(e) The uniformity of the image produced by all array elements. 

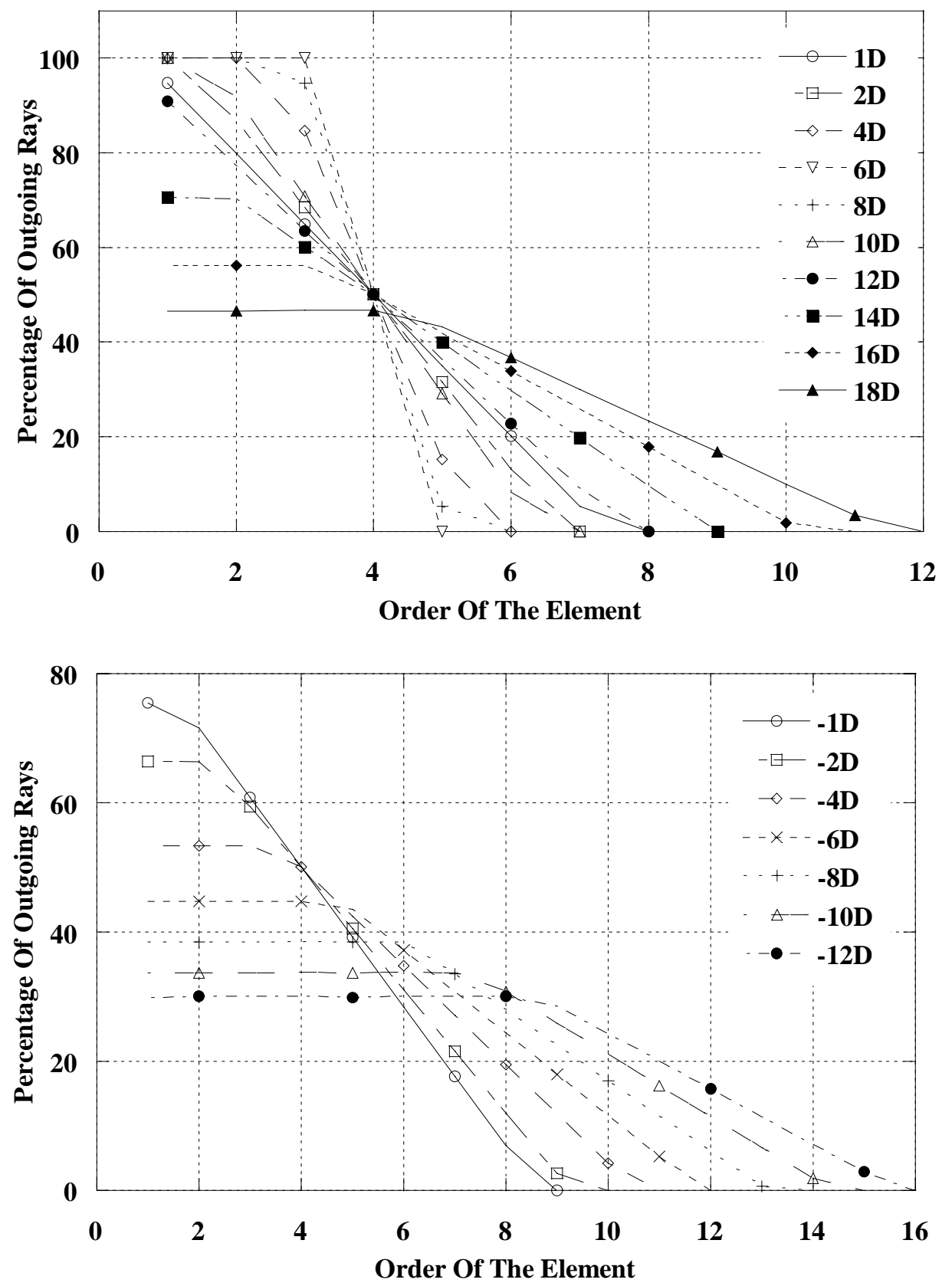

Fig. (4): The order of each array element in PTLA1 versus the percentage of outgoing rays; (a) For positive PTLA1 power values. (b): For negative PTLA1 power values. 
Considering those criterion and with the aid of the obtained results represented in the above figures, two different planer thin-lens light integrator arrays are designed.

i- The first spatial integrator array has been designed with maximum number of identical elements on PTLA1 offering maximum: transmittance, number of outgoing rays with respect to incoming rays, image width, image uniformity and energy on the synthetic image plane (see plot (3)). For this array, PTLA1 has eight optical elements (up and down the array optical axis) each with a power equal to five diopters,

ii- For the second array, the elements in PTLA1 are not identical. It is designed to transfer as much as possible energy onto the synthetic image plane regardless its uniformity. For each optical element in PTLA1, we have considered its individual behavior at a given position (order) with a definite power. Referring to Fig. (4), the thin-lens elements in PTLA1 were chosen to have the powers given in Table (2) corresponding to each element order. The maximum order of the element in this case is eleven. As the optical elements are arranged on both sides of the array general axis, the total number of elements for this array is twenty two elements. Three factors were taken into account in designing such array. First, increasing the maximum number of elements (array area) that can work together to produce the synthetic image. Second, obtaining maximum efficiency. Finally, choosing (as much as possible) long focal length elements on PTLA1 as it offers less spherical aberrations and reduced astigmatism.

Table (2): Power of the thin-lens elements in PTLA1 corresponding to each order. Case (a): array with maximum order of identical elements. Case (b): array with unidentical elements.

\begin{tabular}{|l|l|l|l|l|l|l|l|l|l|l|l|}
\hline Order of the element & 1 & 2 & 3 & 4 & 5 & 6 & 7 & 8 & 9 & 10 & 11 \\
\hline $\begin{array}{l}\text { Power of lenses on } \\
\text { PTLA1. case (a) }\end{array}$ & 5 & 5 & 5 & 5 & 5 & 5 & 5 & 5 & -- & -- & -- \\
\hline $\begin{array}{l}\text { Power of lenses on } \\
\text { PTLA1.case (b) }\end{array}$ & 2 & 4 & 5 & 1 & -6 & -6 & -6 & -6 & -6 & -6 & -6 \\
\hline
\end{tabular}

In order to compare the behavior of the two previously designed light integrator arrays, a developed computer program has been used to calculate the energy distribution onto the synthetic image plane, normalized to maximum value, and then presented in Fig. (5), the position is being normalized to the paraxial size of the image $\Delta$. The coefficient of uniformity (within the image paraxial size), corresponding to each energy distribution, the percentage of the outgoing light-rays and the energy delivered onto the synthetic image plane have been calculated and presented in Table (3). 


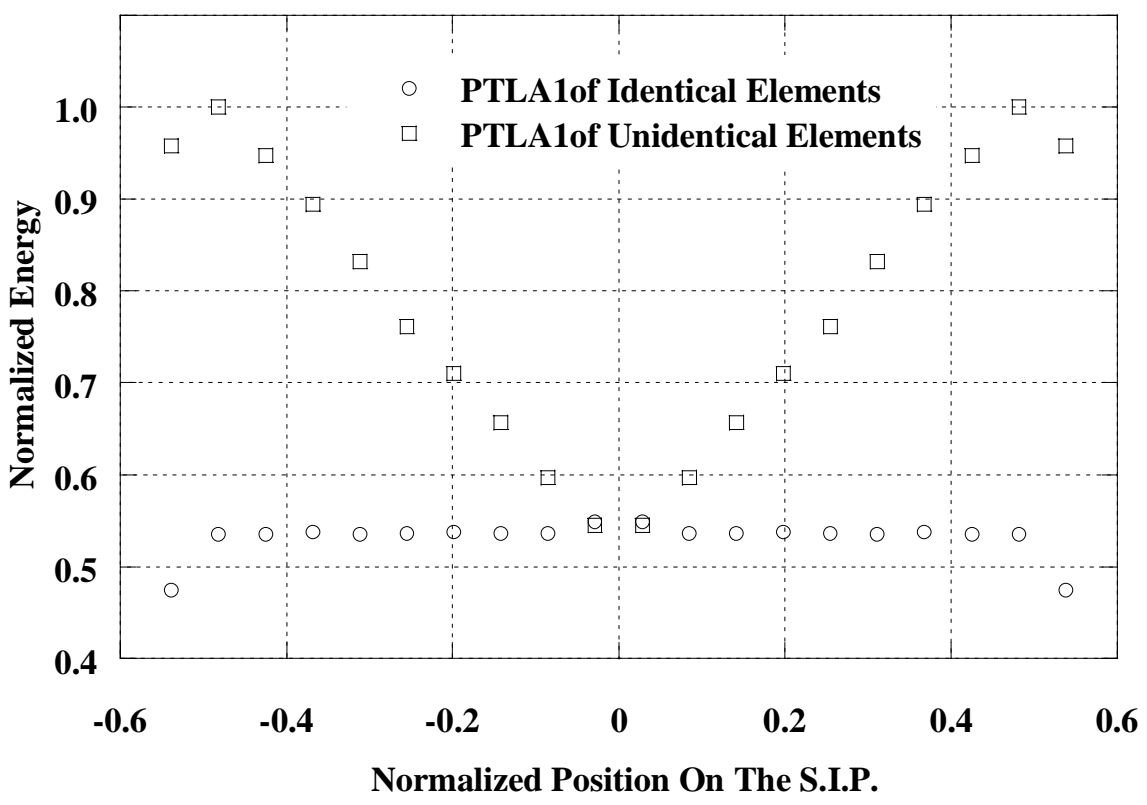

Fig. (5): Energy distribution onto the S.I.P. normalized to the maximum value for two planar thin-lens spatial integrator arrays with identical and unidentical elements of PTLA1.

Table (3): Coefficient of uniformity of the energy distribution onto the S.I.P. and the percentage of outgoing rays of planar thin lens light integrator arrays with (a) identical, (b) unidentical elements. Geometrical parameters are given in table (1), powers of PTLA1 elements are given in Table (2).

\begin{tabular}{|c|c|c|c|}
\hline Case & Coeff. of uniformity $u$ & $\begin{array}{c}\text { Energy on the } \\
\text { S.I.P (Arb.U.) }\end{array}$ & $\begin{array}{c}\text { Percentage of outgoing } \\
\text { rays }\end{array}$ \\
\hline $\mathrm{a}$ & .0769 & 4690.1 & 75 \\
\hline $\mathrm{b}$ & .9097 & 6977.4 & 47.35 \\
\hline
\end{tabular}

\section{Conclusions:}

By studying the behavior of light integrator arrays formed by a couple of planar thin lens arrays, we have found how the type and power of the input array surface influence its; (a) size (maximum number of elements), (b) Efficiency of the array represented by the amount of energy and the percentage of outgoing rays with respect to incoming rays delivered onto the synthetic 
image plane, (c) Synthetic image quality evaluated by image width and coefficient of uniformity. The significance of the previously obtained results is that it enables the design of two different examples of planer thin lens light integrator arrays adequate for different array applications.

\section{Acknowledgment}

This work has been done durant the stay of Dr. Kamal in Optics Department, Universidad Complutense De Madrid. The author would like to thank Dr. Daniel Vazquez for his suggestions and comments about this paper.

\section{References:}

1. Wang Shaomin, Laura Ronchi, Progress in optics, 25, North-Holand, Amsterdam (1988) 279.

2. S. Wang, D. Zhao, "Matrix Optics", Springer-Verlag, Heidelberg”, (2000).

3. JavierAlda, HalaKamal, Eusebio Bernabeu, Opt. Eng. 36, (1997) 2872.

4. Hala Kamal, "design and properties of optical arrays", $\mathrm{PhD}$ dissertation, Universidad Complutense de Madrid, Spain, (1998).

5. Daniel Vázquez, Eusebio Bernabeu, Lig.Res.Tec, 29 (1), (1997) 33.

6. Daniel Vázquez, Javier Alda, and Eusebio Bernabeu, Applied Optics 38, (1999) 1133.

7. H. Kamal, Optik 114, (2003) 213.

8. Hala Kamal, Daniel Vázquez and Javier Alda, Egypt. J. Solids, Vol. 27 (1), (2004) 35. 